\title{
Applying universality in the development of cascade processes for the research of high energy cosmic particles in space experiments
}

\author{
Anastasiya Fedosimova, Pavel Kharchevnikov, Igor Lebedev ${ }^{\mathrm{a}}$, and Abzal Temiraliev \\ Institute of Physics and Technology, Almaty, Kazakhstan
}

\begin{abstract}
An approach for measuring the energy of high-energy particles by a thin calorimeter, is presented. The method is based on the universality in the development of cascade processes. For measurements of the primary energy of cosmic ray particles, the correlation analysis of the dependence of the number of secondary particles, $N_{e}$, at the observation level and the relation of the number of particles, $d N$, at two levels, divided by an absorber layer, is used. It is shown, that the use of correlation curves $\left(\log N_{e}\right.$ versus $\left.d N\right)$ allows to essentially reduce errors in determining the energy of the primary particle, which are connected with the uncertainty in the nature of the primary nucleus and with fluctuations in the development of the cascade process. Uncertainties of energy reconstruction on the basis of the correlation curves methodology, is less than 10 percent.
\end{abstract}

\section{Introduction}

Research of the characteristics of cosmic rays (CR) on the basis of "direct" measurements outside the atmosphere on spacecraft or high-altitude balloons can allow us to solve many problems of particle physics, cosmic ray physics and astrophysics. Consequently, this region has a significant interest and dynamism to the development of theoretical and experimental studies worldwide [1].

Direct experiments on high-altitude balloons, JACEE and RUNJOB, which were aimed to study the elemental composition of cosmic rays, gave very similar spectra of protons, but very different spectra of helium nuclei [2]. Data on heavier nuclei have weak statistics due to the relatively high energy threshold of the applied methodology. Great hopes were pinned on the ATIC [3] and CREAM [4] experiments, however, contradictory data were obtained from the spectral indices of the basic elements of cosmic rays, which do not provide a consistent picture of the processes occurring in the sources of cosmic rays and their propagation to the Earth.

The main advantage of direct experiments is the ability to measure the charge of the incident particle. It is much harder to use an energy detector for particles with energies of $E>10^{12} \mathrm{eV}$ outside the atmosphere.

For particles at relatively low energies, $E<10^{12} \mathrm{eV}$, one can use different techniques such as threshold Cherenkov counters, the method of the RICh detector or the technique of a magnetic mass spectrometer. At higher energies, principal threshold effects, limiting their application, arise. The only best option remaining for energy measurements of different nuclei in a wide energy range (at $E>10^{12} \mathrm{eV}$ ) is, at present, the ionization calorimeter method [5].

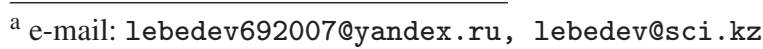

The technical realization of modern ionization calorimeters can be various, but the idea remains invariable: the primary particle enters into a dense substance (for example, iron or lead), in which numerous nuclear and electromagnetic interactions take place giving rise to a cascade of secondary particles.

For measuring the characteristics of the cascade the dense substance is sandwiched between special detectors.

By measuring the signals from these detectors the cascade curve (dependence on the number of particles in the cascade, $N_{e}$, versus penetration depth, $d$, of the cascade in the calorimeter) is formed. If the maximum of a cascade curve is measured, then the energy of a primary particle can be determined.

The main problem with this method of energy measurement is the need for a massive installation (dozens of tons) because the calorimeter must have a sufficiently large depth to build the cascade curve. The huge weight of the installation greatly complicates the possibility of using such a device in space experiments.

In this regard, a more promising approach, for determining the energy on the basis of direct measurements of $\mathrm{CR}$, is the use of a thin calorimeter. In a thin calorimeter the whole cascade of secondary particles is not measured, and only the beginning of the cascade is recorded. The energy is determined on the basis of the analysis of the size of the cascade, because the number of particles in the cascade is almost proportional to the energy of the primary particle [6].

The most interesting example of a thin calorimeter is the detector of the space experiment NUCLEON [7]. To improve the energy reconstruction a combined approach is used: the number of secondary particles in the cascade and additionally their spatial density, are measured.

The accuracy of the energy determination is about 50 percent. The weight of the installation is about $200 \mathrm{~kg}$. 


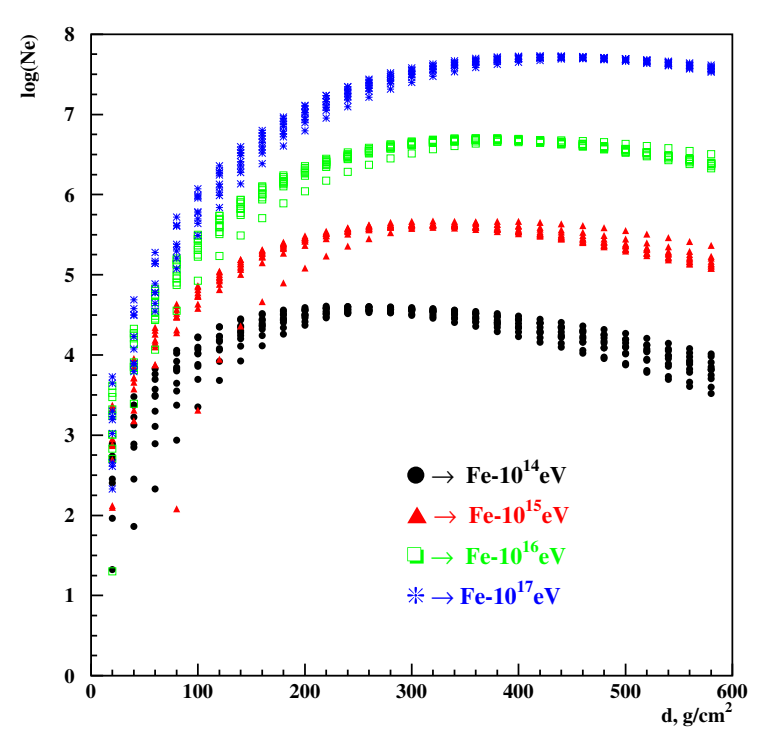

Figure 1. Cascade curves, initiated by iron nuclei at energies $10^{14} \mathrm{eV}, 10^{15} \mathrm{eV}, 10^{16} \mathrm{eV}, 10^{17} \mathrm{eV}$ in air.

In the approach, proposed in our work, the assumption about the universality of the development of the cascade, is used. What is meant?

\section{The method of correlation curves}

The main problem for energy measurements by a thin calorimeter is due to fluctuations of $N_{e}$.

Let's look at Fig. 1, which shows cascade curves, initiated by iron nuclei at energies $10^{14} \mathrm{eV}, 10^{15} \mathrm{eV}$, $10^{16} \mathrm{eV}, 10^{17} \mathrm{eV}$ in air. The showers were simulated using CORSIKA QGSJET [9].

As it is seems from Fig. 1, the cascade curves essentially fluctuate and practically merge (not separable) at small values of penetration depth, d. This fact does not allow us to use them for determining the energy of a primary particle on the basis of a thin calorimeter, i.e., on the basis of the limited quantity of measurements on an ascending branch of a cascade curve.

Most experimental groups define the primary energy, $E$, on the basis of measuring the number of secondary particles, $N_{e}$, at the observation level, using the dependence:

$$
N_{e}=a E^{b}
$$

where the parameters $a, b$, depend on the penetration depth and mass of the primary particle. Statistically the equation works correctly.

However, on the ascending branch of the cascade curve the energy is defined above the real value for quickly developing cascades, and a lower real energy for slowly developing cascades.

What is the reason for such huge fluctuations?

First of all, the development speed of the shower depends on fluctuations of the penetration depth before the first interaction.

In addition, fluctuations in shower development depend on peculiarities of nucleus-nucleus interaction and the initial geometry of the collision. For the research of such peculiarities in multiparticle production, different approaches and methods are used [10-13].

In this paper specific features of nucleus-nucleus interaction are not analyzed. Only the number of particles produced in the first interaction is important.

For example, showers with the multiplicity in the first interaction of $M=100$ particles, develop faster than showers with $\mathrm{M}=10$ particles. After the first interaction, each secondary particle can interact once more. But, when the number of participating particles is larger, the fluctuations in the shower development is less, because fluctuations of individual interactions more or less compensate each other.

The main idea of the approach, proposed in our work, is based on two main positions:

1. The assumption about the universality of the development of the cascade is the following.

Among the various causes of fluctuations, the greatest effect is achieved from fluctuations of the first (first few) interaction.

When the number of participating particles is large, the fluctuations of individual interactions more or less compensate each other. Therefore, all the cascades, formed by primary particles of the same mass and energy, starting from a certain moment, develop almost identically.

This assumption was confirmed by simulation results.

2. The value of the penetration depth is not suitable as a parameter to determine the energy of the primary particles. And so, a new set of correlation parameters must be found which do not depend on fluctuations in the cascade process development.

The influence of fluctuations in cascade development on the results of measurement of the energy can be essentially reduced by using correlation methods of the analysis of the development of the cascade. It allows to considerably increase the energy measurement accuracy.

The method of correlation curves has recommended itself successfully in the analysis of extensive air showers [14].

A modified technique is applied in the given work. The method is developed and tested for the decision of a problem of the measurement of the energy of high-energy particles by thin calorimeters based on computer calculations.

In the technique of correlation curves some internal correlations, which are independent on fluctuations in cascade development, are analyzed.

As a result of the research of the various parameters, characterizing the development of the cascade process, it is revealed that the determination of the energy accuracy can be essentially increased if we use the correlation curve of the dependences of particles number, $\log N_{e}(d 1)$, at the observation level, $d 1$, versus a relation of the number of particles $d N=\log N_{e}(d 1)-\log N_{e}(d 2)$ at two levels, $(d 1$ and $d 2$ ), divided by a layer of an absorber.

In Fig. 2 the correlation curves for the same showers, as in Fig. 1, are presented.

Correlation curves, as seen from the figure, represent a more ordered picture. Fluctuations of an ascending branch 


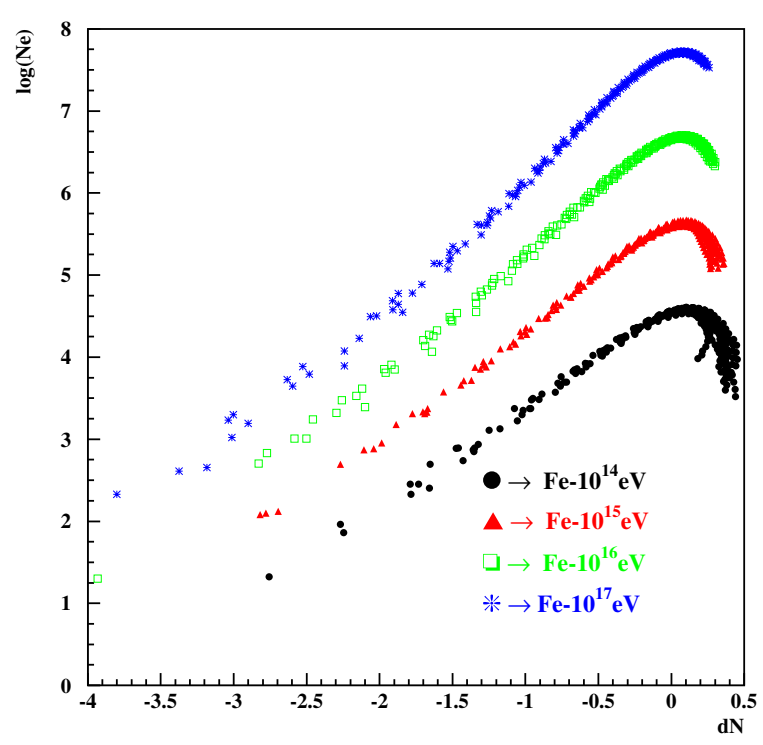

Figure 2. Correlation curves, initiated by iron nuclei at energies $10^{14} \mathrm{eV}, 10^{15} \mathrm{eV}, 10^{16} \mathrm{eV}, 10^{17} \mathrm{eV}$ in air. The thickness of the absorber is $100 \mathrm{~g} / \mathrm{cm}^{2}$.

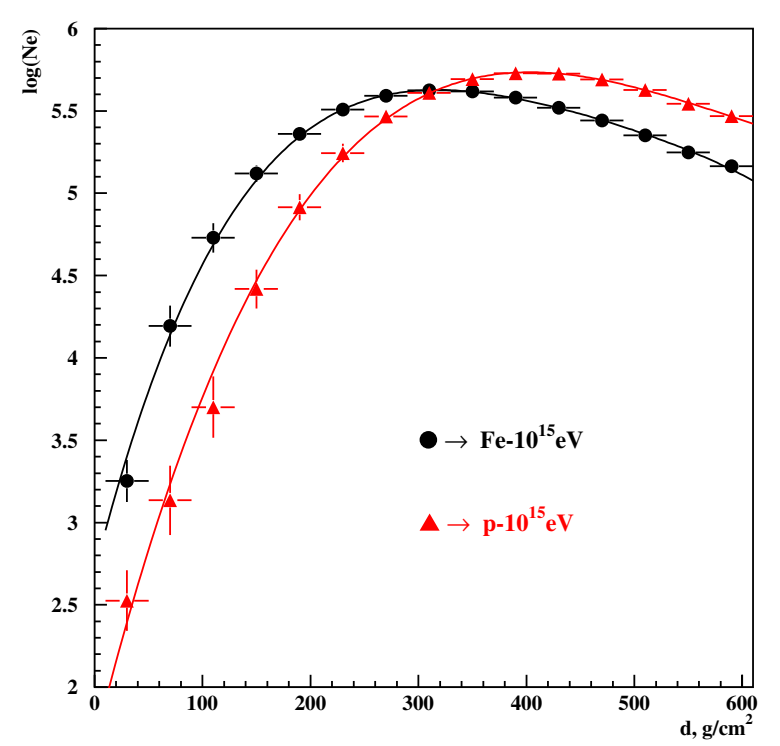

Figure 3. Average cascade curves, initiated iron nuclei and proton at energy $10^{16} \mathrm{eV}$ in the air.

of a correlation curve are not as considerable, as in the case of cascade curves.

Figures 1 looks like a mess at the beginning of the cascade curves but Fig. 2 shows a rather ordered structure in the correlation curves. The negative region of $d N$ corresponds to small values of the penetration depth.

The second most important parameter, influencing the accuracy of the energy measurement, is the uncertainty of the nature of the primary nucleus.

In Fig. 3 average cascade curves of interactions of iron nuclei and protons at a fixed energy $10^{16} \mathrm{eV}$ with the nuclei of air atoms, are presented.

The cascade curves, formed by a proton, are shifted in area of the bigger depths of penetration in comparison with Fe cascade curves. This fact leads to underestimating the energy of proton cascades and to an overestimation for Fe cascades.

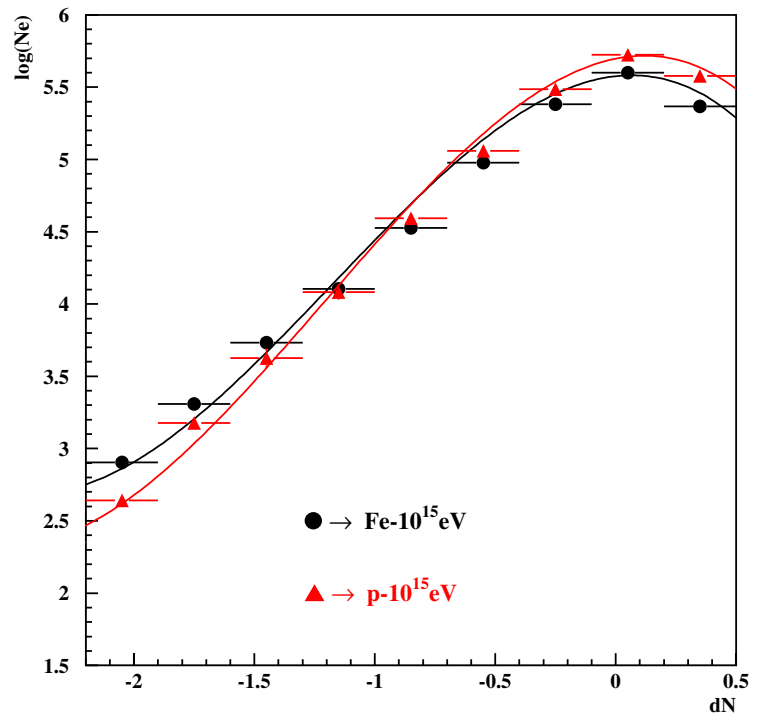

Figure 4. Average cascade curves, initiated by iron nuclei and protons at energy $10^{16} \mathrm{eV}$ in air. The thickness of the air absorber is $100 \mathrm{~g} / \mathrm{cm}^{2}$.

Using correlation curves allows to essentially reduce energy definition errors, connected with the uncertainty of the primary nucleus.

Average correlation curves are presented in Fig. 4. As seen from the figure, correlation curves practically coincide for different nuclei. Maximum points of proton and iron correlation curves are shifted in one point $d N=$ 0 , which is independent from the depth of penetration.

Summing up the given section we once again underline the following: the accuracy of the energy definition on the basis of a thin calorimeter can be essentially increased if we use the correlation curve dependences of the number of particles at the observation level versus a difference of the number of particles at the two levels, divided by an absorber layer.

\section{Procedure of the energy reconstruction}

The analysis procedure consisted of several basic stages:

1. The simulation of cascades, formed by primary protons and iron nuclei with fixed energies.

2. Fitting the $\log N-d N$ distributions by polynomial functions of the third order for each fixed energy as a function of $\log N$ versus $d N$ in the form:

$$
\log N(d N)=a_{0}+a_{1} d N+a_{2} d N^{2}+a_{3} d N^{3}
$$

3. Fitting the coefficients of $a_{0}, a_{1}, a_{2}, a_{3}$ in the dependence on the energy and the construction of the dependence $\log N$ on $d N$ and $E_{0}$ in the form:

$$
\begin{aligned}
\log N\left(d N, E_{0}\right)=a_{0}\left(E_{0}\right) & +a_{1}\left(E_{0}\right) d N \\
& +\ldots+a_{3}\left(E_{0}\right) d N^{3}
\end{aligned}
$$

4. The simulation of test cascades, formed by primary protons and iron nuclei with arbitrary energies, and reconstruction of the primary energy on the basis of the dependence of Eq. (3). 
To determine the energy of $i$-th test cascade, using a set of curves (Eq. (3)), it is necessary to vary $d N$ and $E_{0}$ so that at the same time minimize the following differences

$$
\begin{gathered}
\left|\log N_{m}-\log N\left(d N, E_{0}\right)\right|<\epsilon \\
\left|d N_{m}-d N\right|<\epsilon
\end{gathered}
$$

where $\log N_{m}$ and $d N_{m}$ are "measured" characteristics of the test cascade at the observation level.

By the definition of the energy of the test cascades it was found that uncertainties in the energy reconstruction, $<\left|\left(E_{i}-E_{0}\right) / E_{0}\right|>$, in the energy estimation by the formulae $2-5$, is less than 10 percent.

The accuracy of the energy determination by the standard technique of cascade curves, in the case of registration of the cascade to the depth of its maximum development, is about 50 percent [6].

This clearly argues in favor of the proposed methodology for determining the primary energy, as it significantly reduces the required thickness of the calorimeter and improves the accuracy of the measurements.

\section{Conclusion}

An approach for measuring the energy of high-energy particles by a thin calorimeter, is presented.

It is shown, that the use of correlation curves $\left(\log N_{e}\right.$ versus $d N$ ) allows to essentially reduce the errors in the determination of the primary particle energy, which are connected with the uncertainty in the nature of the primary nucleus and with fluctuations in the development of the cascade process.

Uncertainties of energy reconstruction on the basis of the correlation curves methodology, is less than 10 percent.

Further, for a possible technical realization of the project of a thin calorimeter it will be necessary to solve problems connected with the calculation of the installation response, the estimation of the influence of the interaction model on the reconstruction results, etc.
However, now the main result is received: on the basis of computer simulation the correlation parameters, which are independent on fluctuations in shower development and which are almost independent on the uncertainty of the primary nucleus, allow us to determine the energy of the primary nuclei with very high accuracy for thin calorimeters.

\section{References}

[1] R. Sparvoli, Nucl. Phys. B - Proc. Suppl. 239-240, 115-121 (2013)

[2] E.-S. Seo, Astropart. Phys. 76, 29-40 (2012)

[3] H.S. Ahn et al., Ap.J. 714, 89-93 (2010)

[4] D. Panov, N.V. Sokolskaya, V.I. Zatsepin, JP: Conf.Ser. 409, 012036 (2013)

[5] M.I. Panasyuk et al., The mystery of the origin of galactic cosmic rays Bull. Moscow Univ., ser.3 6, 25-30 (2012)

[6] V.S. Murzin, Astrophysics of cosmic rays (MGU, Moscow, 2006) 668p.

[7] V.L. Bulatov at al., Testing of technological prototype of «NUCLEON» installation on the pion beam PTE 1, 34-41 (2010)

[8] D. Heck, J. Knapp, J.N. Capdeville, G. Schatz, T. Thouw, CORSIKA: A Monte Carlo Code to Simulate Extensive Air Showers (FZKA 6019, Karlsruhe, 1998) 89p.

[9] M.I. Adamovich et al., Azimutal correlations of secondary particles in ${ }^{32} S$ induced interactions with $\mathrm{Ag}(\mathrm{Br})$ nuclei at $4.5 \mathrm{GeV/c/nucleon} \mathrm{Part.} \mathrm{and} \mathrm{Nucl.}$ Lett. 101, 76 (2000)

[10] M.I. Adamovich et al., Factorial Moments of ${ }^{28} \mathrm{Si}$ Induced Interactions with $\mathrm{Ag}(\mathrm{Br})$ Nuclei APH N.S. Heavy Ion Phys. 113213 (2001)

[11] I.A. Lebedev et al., J. Phys. G 23, 637 (1997)

[12] T.N. Kvochkina et al., J. Phys. G 26, 35 (2000)

[13] A.I. Fedosimova et al., J. Phys.: Conf. Ser. 668, 012067 (2016)

[14] W.D. Apel et al., Applying Shower Development Universality to KASCADE data Astropart.Phys. 29, N6, 412-419 (2008) 